PROCEEDINGS OF THE

AMERICAN MATHEMATICAL SOCIETY

Volume 130, Number 7, Pages 2049-2055

S 0002-9939(02)06350-5

Article electronically published on January 17, 2002

\title{
ON NUMERICAL BLOW-UP SETS
}

\author{
JULIÁN FERNÁNDEZ BONDER, PABLO GROISMAN, AND JULIO D. ROSSI
}

(Communicated by David S. Tartakoff)

\begin{abstract}
In this paper we study numerical blow-up sets for semidicrete approximations of the heat equation with nonlinear boundary conditions. We prove that the blow-up set either concentrates near the boundary or is the whole domain.
\end{abstract}

\section{INTRODUCTION}

In this paper we address the localization problem of blow-up points for semidiscretizations in the space of positive solutions for the following parabolic problem:

$$
\begin{aligned}
u_{t}(x, t) & =u_{x x}(x, t), & & (x, t) \in(0,1) \times[0, T), \\
u_{x}(1, t) & =f(u(1, t)), & & t \in[0, T), \\
u_{x}(0, t) & =0, & & t \in[0, T), \\
u(x, 0) & =\varphi(x), & & x \in[0,1],
\end{aligned}
$$

where $f(s)$ and $\varphi(x)$ are positive and regular enough to guarantee existence and uniqueness of solutions. For this type of problem, if the initial datum satisfies a compatibility condition, the solution is smooth (see [2, 10]).

The purpose of this article is to show that blow-up sets for semidiscrete approximations of (11) (by the finite element method) either concentrate near the boundary or are the whole $[0,1]$; that is, regional blow-up does not exists for these schemes.

Parabolic reaction-diffusion problems like (11) or of a more general form, allowing for example source terms or with different boundary conditions, appear in several branches of applied mathematics. They have been used to model, for example, chemical reactions, heat transfer, or population dynamics, and have been studied by several authors. See $[12$ and the references therein.

For many differential equations or systems the solutions can become unbounded in finite time (a phenomenon that is known as blow-up). Typical examples where this phenomenon is observed are problems involving reaction terms in the equation (see for example [9], [12]). When the nonlinear term appears at the boundary, as

Received by the editors September 19, 2000 and, in revised form, February 7, 2001.

2000 Mathematics Subject Classification. Primary 35K55, 35B40, 65M20.

Key words and phrases. Numerical approximations, nonlinear boundary conditions.

This work was partially supported by Universidad de Buenos Aires under grants TX47 and TX48 and by ANPCyT PICT No. 03-00000-00137. The third author was also supported by Fundación Antorchas. 
in (11), it is known that if $f$ is convex and satisfies

$$
\int^{+\infty} \frac{1}{f(s) f^{\prime}(s)} d s<+\infty
$$

one has this blow-up phenomenon (see [13). Moreover, if $f$ is increasing and

$$
\int^{+\infty} \frac{1}{f(s)} d s<+\infty
$$

then the blow-up set is localized at the boundary. In this case $B(u)=\{1\}$, which is called single point blow-up.

However, when $f$ satisfies (2) but not (3), the blow-up set can be larger than a single point. It can be the whole interval $[0,1]$ (this is called global blow-up), or it can be a subinterval $[l, 1]$ with $0<l<1$ (this is called regional blow-up). For example, this phenomenon of global blow-up or regional blow-up is obtained when one considers $f(s)=s \log ^{p}(s)$ with $1 / 2<p<1$ or $p=1$ respectively.

On the other hand, some papers deal with the numerical approximation of the blow-up time and the blow-up profile (see for example the survey [3] and the work 4]), but there is a lack of information about numerical blow-up sets.

Now we describe our numerical scheme. The numerical semidiscrete version of (11) proposed here, a first degree finite element approximation on the variable $x$ keeping $t$ continuous, is well known to coincide with a classical finite difference second order scheme on $x$. The mass lumping technique simplifies the scheme and preserves the maximum principle, allowing us to use comparison arguments (see Lemma 10.

Let $x_{i}=\frac{i}{N}, 0 \leq i \leq N$, be a partition of the interval $[0,1]$ into subintervals $I_{i}=$ $\left[x_{i}, x_{i+1}\right]$, of length $h=\frac{1}{N}$. Let $V_{h}$ the set of continuous functions which are affine on each $I_{i}$. We consider the basis functions of $V_{h}$, taking as usual $\psi_{i}$, with $\psi_{i}\left(x_{j}\right)=\delta_{i}^{j}$. Now let

$$
u_{h}(x, t)=\sum_{i=0}^{N} u_{i}(t) \psi_{i}(x) .
$$

For a fixed $t, u_{h}(x, t)$ belongs to $H^{1}(0,1)$, so in order to construct an approximate solution of (1) we proceed as follows: replacing (4) in the weak formulation of (1), we get a system of ordinary differential equations for $U=\left(u_{0}(t), \ldots, u_{N}(t)\right)$ :

$$
M U^{\prime}=-A U+f(U)
$$

where $M$ is the mass matrix, $A$ is the stiffness matrix, and $f(U)=f\left(u_{N}\right) e_{N}$ with $e_{N}=(0, \ldots, 1)$.

Mass lumping upon the matrix $M$ in (5) gives

$$
\begin{aligned}
u_{0}^{\prime}(t) & =\frac{2}{h^{2}}\left(-u_{0}(t)+u_{1}(t)\right), \\
u_{i}^{\prime}(t) & =\frac{1}{h^{2}}\left(u_{i+1}(t)-2 u_{i}(t)+u_{i-1}(t)\right), \\
u_{N}^{\prime}(t) & =\frac{2}{h^{2}}\left(-u_{N}(t)+u_{N-1}(t)\right)+\frac{2}{h} f\left(u_{N}(t)\right) .
\end{aligned}
$$

So, from now on, we will assume that $M$ is diagonal with $m_{i}=h$ if $1 \leq i \leq N-1$ and $m_{0}=m_{N}=h / 2$. 
For this scheme it is known that $u_{h}$ converges uniformly to $u$ provided that $u$ is smooth, see [5]. Also it is proved there that solutions blow up if and only if $f$ satisfies (3), so there exist nonlinearities $f$ such that the continuous solution blows up while the numerical solutions does not (for example for $f(s)=s \ln (s)^{p}$ with $1 / 2<p \leq 1)$.

In 1] the authors studied numerical approximations for (1) in the special case $f(s)=s^{p}$, proving that numerical blow-up sets approximate the continuous one when the mesh parameter $h$ is small. See also 8] for a similar result in the semilinear case.

In this work, we show that regional blow-up can never be reproduced for our scheme, and this is a big difference in the behavior between solutions of (1) and their numerical approximations that was never pointed out before.

The difference in the asymptotic behavior between solutions of differential equations and their numerical approximations has been observed by several authors in recent years (see for example [6], [7]) and is a phenomenon that has to be taken into consideration when performing simulations.

\section{STATEMENT OF THE RESUlts}

Now we can state our main results:

Theorem 1. Assume that $f$ satisfies (3) and is increasing. Then, the number of blow-up nodes for solutions of (6) depends only on $f$ and is independent of $h$. That is, either the numerical blow-up is global or it is localized in a small neighborhood of $\{x=1\}$ for $h$ small enough. Moreover, the propagation of blow-up is given by the following mechanism:

Let $T_{h}$ be the blow-up time of $U$ and let

$$
\mathcal{F}(z)=\int_{z}^{+\infty} \frac{1}{f(y)} d y ; \quad G_{0}(t)=\mathcal{F}^{-1}\left(T_{h}-t\right) ; \quad G_{k+1}(t)=\int_{0}^{t} G_{k}(s) d s .
$$

Then $u_{N-k}$ blows up if and only if $G_{k}$ does, and in this case the blow-up rate is given by

$$
u_{N-k}(t) \sim \int_{0}^{t} u_{N-k+1}(s) d s .
$$
$f$.

Also we prove the monotonicity of the blow-up set in terms of the nonlinearity

Theorem 2. Under the hypotheses of Theorem 1, the number of blow-up nodes for solutions of (6) is monotone decreasing with the nonlinear term $f$. That is, if $f_{1}(s) \geq f_{2}(s)$, then $B_{h}\left(U_{1}\right) \subset B_{h}\left(U_{2}\right)$.

Finally we show that the blow-up rate of the blowing up nodes is strictly decreasing.

Theorem 3. The blow-up rate for the blowing up nodes $x_{i}$ is strictly monotone in the sense that

$$
\lim _{t \nearrow T_{h}} \frac{u_{i}(t)}{u_{i+1}(t)}=0
$$


Let us now present some examples.

(I) $f(s)=s^{p}$.

Numerical solutions blow up if and only if $p>1$. The blow-up rate for different nodes is given by $u_{N-k}(t) \sim\left(T_{h}-t\right)^{-1 /(p-1)+k}$ for $0 \leq k<1 /(p-1)$. If $1 /(p-1)=$ $k$, an integer, then the node $u_{N-k} \sim-\ln \left(T_{h}-t\right)$. The blow-up set is composed of exactly $K$ nodes, where $K=[1 /(p-1)]([x]$ stands for the integer part of $x)$.

In this case, the blow-up condition $p>1$ is the same as in the continuous problem (1), and the blow-up set satisfies

$$
B\left(u_{h}\right)=[1-K h, 1]=B(u)+[-K h, 0] .
$$

This case has been considered in [1].

(II) $f(s)=s(\ln s)^{p}$.

Numerical solutions blow up if and only if $p>1$. The blow-up rate is given by

$$
\max _{i} u_{i}(t) \sim \exp \left(\frac{1}{\left(T_{h}-t\right)^{1 /(p-1)}}\right) .
$$

As a consequence of (I) and Theorem 2 the blow-up set is the whole interval $[0,1]$.

In this case, the blow-up condition $p>1$ is different from the condition in the continuous problem (11), $p>1 / 2$. The blow-up set for the continuous problem is a single point, $B(u)=\{1\}$, if $p>1$; a bounded interval if $p=1$; and the whole $[0,1]$ if $1 / 2<p<1$. In this case, we remark that the behavior of the continuous problem is radically different from the numerical one, since not only do we have different blow-up cases, but also in the cases where both problems blow up, the numerical solution has global blow-up while the continuous problem has single-point blow-up. This is the first example of such phenomena.

(III) $f(s)=e^{s}$.

In this case numerical solutions blow up at a single point $B\left(u_{h}\right)=\{1\}$. The blow-up rate is given by $u_{N}(t) \sim-\ln \left(T_{h}-t\right)$.

For this nonlinearity, the blow-up sets and the blow-up rates of both problems (the continuous and the discrete ones) coincides.

\section{SOME TECHNICAL LEMMAS}

In order to study the asymptotic behaviour of (6) we need the following results.

Lemma 1 (Maximum principle). Let $h>0$ be fixed, and let $U=\left(u_{0}, \ldots, u_{N}\right)$ be $a$ solution of

$$
\begin{aligned}
u_{0}^{\prime} & \leq \frac{2}{h^{2}}\left(-u_{0}+u_{1}\right), \\
u_{i}^{\prime} & \leq \frac{1}{h^{2}}\left(u_{i+1}-2 u_{i}+u_{i-1}\right), \\
u_{N}^{\prime} & \leq \frac{2}{h^{2}}\left(-u_{N}+u_{N-1}+h f\left(u_{N}\right)\right) .
\end{aligned}
$$

Then

$$
\max _{k=0, \ldots, N} u_{k}(t) \leq \max \left\{\max _{k=0, \ldots, N} u_{k}(0) ; \sup _{0<\tau<t} u_{N}(\tau)\right\}
$$

Proof. Let us first suppose that $U=\left(u_{0}, \ldots, u_{N}\right)$ satisfies (7) with strict inequalities. Now, if the maximum is attained in an interior node, say $0<j<N$, let $t_{0}$ be the 
first time when this happens; then we have

$$
u_{j}^{\prime}\left(t_{0}\right) \geq 0 \quad \text { and } \quad u_{j}\left(t_{0}\right) \geq u_{k}\left(t_{0}\right), \quad 0 \leq k \leq N .
$$

On the other hand, by our assumption on $U$ we get $u_{j}^{\prime}\left(t_{0}\right)<0$, which leads to a contradiction. Also, it is easy to see that the maximum cannot be reached at $u_{0}$, and so the "maximum principle" follows.

To complete the proof, we just observe that if $Z=h^{2}\left(0, \ldots, k^{2}, \ldots, N^{2}\right)$, then $U_{\varepsilon}(t) \equiv U(t)+\varepsilon Z$ satisfies (7) with strict inequalities whenever $U$ satisfies (7). As $\varepsilon>0$ is arbitrary, the lemma follows.

Lemma 2. Let $U$ be a blowing-up solution of (6). Then there exists a time $t_{0}<T_{h}$ such that

$$
\max _{0 \leq i \leq N} u_{i}(t)=u_{N}(t)
$$

for $t_{0}<t<T_{h}$.

Proof. First, observe that from our assumptions on $f$, it follows that

$$
\lim _{s \rightarrow+\infty} \frac{f(s)}{s}=+\infty
$$

Now, by the maximum principle, it follows that if $\max \left\{u_{i}(t)\right\}$ is large then it coincides with $\sup _{0<s<t} u_{N}(s)$. Now, from (6) it follows that

$$
u_{N}^{\prime} \geq-\frac{2}{h^{2}} u_{N}+\frac{2}{h} f\left(u_{N}\right) \geq \frac{2}{h} f\left(u_{N}\right)\left(1-\frac{u_{N}}{h f\left(u_{N}\right)}\right) .
$$

As $u_{N}(t)$ is blowing up and (9) holds, $u_{N}(t)$ is an increasing function for $t$ near the blow-up time $T_{h}$, and hence we conclude that (8) holds for $t$ close to $T_{h}$.

\section{PROOF OF THE THEOREMS}

In this section we denote by $C$ a constant that may depend on $h$ but not on $t$ and may change from one line to another. Let us begin with the proof of Theorem [1. For the proof, we need the following lemma.

Lemma 3. There exists a constant $C$ (that may depend on $h$ ) such that the blow-up rate for $u_{N}(t)$ (and hence for $U(t)$ ) is given by

$$
\max _{k} u_{k}(t)=u_{N}(t) \sim \mathcal{F}^{-1}\left(C\left(T_{h}-t\right)\right),
$$

where $\mathcal{F}(z)$ is defined as

$$
\mathcal{F}(z)=\int_{z}^{+\infty} \frac{1}{f(y)} d y .
$$

Proof. Integrating (10), we obtain

$$
u_{N}(t) \leq c_{1} \mathcal{F}^{-1}\left(C\left(T_{h}-t\right)\right)
$$

For the other inequality, we observe that for $t$ close to $T_{h}$, by Lemma 2, we obtain

$$
u_{N}^{\prime}(t)=\frac{2}{h^{2}}\left(u_{N-1}-u_{N}\right)+\frac{2}{h} f\left(u_{N}\right) \leq \frac{2}{h} f\left(u_{N}\right) .
$$

Hence by integrating this inequality we get

$$
u_{N}(t) \geq \mathcal{F}^{-1}\left(C\left(T_{h}-t\right)\right)
$$

and the lemma follows. 
Proof of Theorem 1. Now, using the preceding lemma, we look for the behaviour of the node $u_{N-1}$,

$$
u_{N-1}^{\prime}(t)=\frac{u_{N}(t)-2 u_{N-1}(t)+u_{N-2}(t)}{h^{2}} \geq \frac{1}{h^{2}}\left(u_{N}(t)-2 u_{N-1}(t)\right) .
$$

So,

$$
\left(e^{\frac{2 t}{h^{2}}} u_{N-1}(t)\right)^{\prime} \geq \frac{e^{\frac{2 t}{h^{2}}}}{h^{2}} u_{N}(t)
$$

and integrating we get

$$
u_{N-1}(t) \geq C \int_{t_{0}}^{t} u_{N}(s) d s+C .
$$

On the other hand, by (8) we get

$$
u_{N-1}^{\prime}(t) \leq \frac{2}{h^{2}}\left(u_{N}(t)-u_{N-1}(t)\right)
$$

and therefore

$$
u_{N-1}(t) \leq C \int_{t_{0}}^{t} u_{N}(s) d s+C
$$

Using Lemma 3, we get

$$
u_{N-1}(t) \sim \int_{t_{0}}^{t} u_{N}(s) d s \sim \int_{t_{0}}^{t} \mathcal{F}^{-1}\left(C\left(T_{h}-s\right)\right) d s .
$$

Recall that $G_{0}(t)=\mathcal{F}^{-1}\left(T_{h}-t\right)$, and

$$
G_{1}(t)=\int_{t_{0}}^{t} \mathcal{F}^{-1}\left(T_{h}-t\right) d s=\int_{T_{h}-t}^{T_{h}-t_{0}} \mathcal{F}^{-1}(w) d w .
$$

Since

$$
\int_{t_{0}}^{t} \mathcal{F}^{-1}\left(C\left(T_{h}-s\right)\right) d s=\int_{C\left(T_{h}-t\right)}^{C\left(T_{h}-t_{0}\right)} \mathcal{F}^{-1}(w) d w,
$$

we have that $u_{N-1}(t)$ blows up if and only if $G_{1}(t)$ does. Repeating this procedure, one can check that $u_{N-k}$ blows up if and only if $G_{k}(t)$ does, so the number of nodes that blow up depends only on $f$ but not on $h$. This fact implies the desired result.

Proof of Theorem 2. To prove Theorem 2 we just have to observe that, if we set $\mathcal{F}_{i}(z)=\int_{z}^{+\infty} \frac{1}{f_{i}(y)} d y, i=1,2$, then we have that

$$
f_{1}(s) \geq f_{2}(s) \Rightarrow \mathcal{F}_{1}(z) \leq \mathcal{F}_{2}(z)
$$

and the result follows.

Proof of Theorem 3. Finally, we prove Theorem 3. First we consider $\frac{u_{N}(t)}{u_{N-1}(t)}$. From the asymptotic behavior found in Theorem 1 , we have

$$
\frac{u_{N}(t)}{u_{N-1}(t)} \sim \frac{u_{N}(t)}{\int_{t_{0}}^{t} u_{N}(s) d s} \text {. }
$$


Now by L'Hôpital's rule and (9), we obtain

$$
\lim _{t \nearrow T_{h}} \frac{u_{N}(t)}{\int_{t_{0}}^{t} u_{N}(s) d s}=\lim _{t \nearrow T_{h}} \frac{u_{N}^{\prime}(t)}{u_{N}(t)}=\lim _{t \nearrow T_{h}} \frac{C f\left(u_{N}(t)\right)}{u_{N}(t)}=+\infty,
$$

and the result follows by induction.

\section{ACKNOWLEDGMENTS}

We want to thank the referee for his or her care in reading the manuscript and pointing out a mistake.

\section{REFERENCES}

[1] G. Acosta, J. Fernández Bonder, P. Groisman and J.D. Rossi. Numerical approximation of the heat equation with nonlinear boundary conditions in several space dimensions. Preprint.

[2] H. Amann. Parabolic evolution equations and nonlinear boundary conditions, J. Diff. Eq. 72 (1988), 201-269. MR 89e:35066

[3] C. Bandle and H. Brunner. Blow-up in diffusion equations: a survey. J. Comp. Appl. Math. 97 (1998), 3-22. MR 99g:35061

[4] Y.G. Chen. Asymptotic behaviours of blowing up solutions for finite difference analogue of $u_{t}=u_{x x}+u^{1+\alpha}$. J. Fac. Sci. Univ. Tokyo, Sec IA, Math. Vol. 33, (1986), 541-574. MR 88a:65113

[5] R. G. Duran, J. I. Etcheverry and J. D. Rossi. Numerical approximation of a parabolic problem with a nonlinear boundary condition. Discr. Cont. Dyn. Sys. 4 (3), 497-506, (1998). MR 99a:65122

[6] C.M. Elliot and A.M. Stuart. Global dynamics of discrete semilinear parabolic equations. SIAM J. Numerical Anal. 30 (1993), 1622-1663. MR 94j:65127

[7] J. Fernández Bonder and J.D. Rossi. Blow-up vs. spurious steady solutions. Proc. Amer. Math. Soc. 129 (1), 139-144, (2001). MR 2001c:35105

[8] P. Groisman and J.D. Rossi. Asymptotic behaviour for a numerical approximation of a parabolic problem with blowing up solutions. J. Comp. Appl. Math. 135 (1), 135-155, (2001).

[9] K. Deng and H. Levine. The role of critical exponents in blow-up theorems: the sequel. J. Math. Anal. Appl. 243, 85-126, (2000). MR 2001b:35031

[10] J. Lopez Gomez, V. Marquez and N. Wolanski. Blow up results and localization of blow up points for the heat equation with a nonlinear boundary condition. J. Diff. Eq. 92(2), 384-401, (1991). MR 92j:35098

[11] D. F. Rial and J. D. Rossi. Blow up results and localization of blow up points in an ndimensional smooth domain. Duke Math. J. 88 (2), 391-405, (1997). MR 98i:35079

[12] C. V. Pao. Nonlinear parabolic and elliptic equations. Plenum Press, 1992. MR 94c:35002

[13] W. Walter. On existence and nonexistence in the large of solutions of parabolic differential equations with a nonlinear boundary condition. SIAM J. Math. Anal. 6(1), 85-90, (1975). MR 51:1122

Departamento de Matemática, FCEyn, ubA (1428) Buenos Aires, Argentina

E-mail address: jfbonder@dm.uba.ar

Departamento de Matemática, FCeyn, UBA (1428) Buenos Aires, Argentina

E-mail address: pgroisma@dm.uba.ar

Current address: Departamento de Matemática y Ciencias, Universidad de San Andrés, Vito

Dumas 284 (B1D1644)-Victoria, Buenos Aires, Argentina

E-mail address: pablog@udesa.edu.ar

Departamento de Matemática, FCEyn, UBA (1428) Buenos Aires, Argentina

E-mail address: jrossi@dm.uba.ar 